\title{
Relações entre evasão, satisfação com escolha profissional, renda e adaptação de universitários
}

\author{
Rodolfo Augusto Matteo Ambiel' \\ Universidade São Francisco, USF, SP, Brasil \\ Leonardo de Oliveira Barros \\ Universidade São Francisco, USF, SP, Brasil
}

\begin{abstract}
Resumo: Esta pesquisa teve como objetivo verificar correlações entre a adaptação acadêmica e os motivos para evasão do ensino superior, com base no controle das variáveis renda e satisfação com a escolha profissional. Utilizaram-se a Escala de Motivos de Evasão no Ensino Superior (M-ES) e o Questionário de Adaptação ao Ensino Superior (QAES), aplicados em 198 universitários de instituições públicas e privadas brasileiras, $85,4 \%$ do sexo feminino, com idade média de 20,92 anos. Constatou-se que estudantes com clareza do projeto de carreira têm menos motivos vocacionais para evasão e que alunos mais adaptados social e emocionalmente apresentam menores motivos para evadir em função do desempenho acadêmico. Verificou-se que a satisfação com a escolha está relacionada à facilidade de adaptação e ao melhor desempenho e que a renda tende a potencializar ou minimizar os motivos para evasão de acordo com o suporte financeiro e social recebido.
\end{abstract}

Palavras-chave: adaptação acadêmica; evasão universitária; escolha profissional; orientação de carreira; avaliação psicológica.

\section{RELATIONS BETWEEN DROPOUT, SATISFACTION WITH PROFESSIONAL CHOICE, INCOME AND ADAPTATION OF UNIVERSITY STUDENTS}

\begin{abstract}
This research aimed to verify correlations between academic adaptation and reasons for avoidance of higher education, controlling for income and satisfaction with professional choice variables. It was used the Estimates for Evasion of Higher Education (M-ES) and the Questionnaire for Adaptation to Higher Education (QAES) in 198 university students of public and private institutions in Brazil, $85.4 \%$ female, with a mean age of 20.92 years. It has been found that students with clear career design have fewer vocational reasons for avoidance and that more socially and emotionally well-off students have fewer reasons to evade due to academic performance. It was observed that the satisfaction with the choice is related to the ease of adaptation and better performance and that the income tends to potentiate or minimize the reasons for evasion according to the financial and social support received.
\end{abstract}

Keywords: academic adaptation; dropout university; professional choice; career counseling; psychological evaluation.

\section{RELACIONES ENTRE EVASIÓN, SATISFACCIÓN CON ELECCIÓN PROFESIONAL, INGRESO Y ADAPTACIÓN DE UNIVERSITARIOS}

Resumen: Esta investigación tuvo como objetivo determinar las correlaciones entre la adaptación académica y las razones de la evasión de la educación superior mediante el

${ }^{1}$ Endereço de correspondência: Rodolfo Augusto Matteo Ambiel: R. Waldemar César da Silveira, 105, Vl, Cura D'Ars (Swift), Campinas, São Paulo, Brasil. CEP: 13045-510. E-mail: rodolfo.ambiel@usf.edu.br 
control de las variables de la renta y la satisfacción con la elección de carrera. Utilizamos las razones van Evasión de la Educación Superior y (M-ES) el Cuestionario de Adaptación a la Educación Superior (QAES) 198 alumnos de público brasileño y las instituciones privadas, el $85,4 \%$ mujeres, con una edad media de 20,92 años. Se encontró que los estudiantes con proyecto de carrera claramente tiene razones menos profesional para la evasión y los estudiantes más aptos sociales y emocionalmente presente menos razones para escapar debido al rendimiento académico. Se constató que la satisfacción con la elección está relacionada con la facilidad de adaptación y mejor desempeño y que la renta tiende a potenciar o minimizar los motivos de evasión de acuerdo con el soporte financiero y social recibido.

Palabras clave: adaptación académica; evasión de la universidad; elección profesional; orientación profesional; evaluación psicológica.

\section{Introdução}

Nos últimos 20 anos, o Brasil evidenciou um crescimento em relação ao acesso ao ensino superior (ES), mediante políticas públicas para inserção de jovens no contexto universitário e de ações afirmativas que permitam o rompimento com situações excludentes que limitam o desenvolvimento educacional especialmente por indivíduos de camadas econômicas mais baixas (Ministério da Educação, 2013). Todavia, para além do crescimento do número de pessoas com acesso ao ES, também há uma quantidade expressiva de universitários que se evadem dos cursos, seja na modalidade presencial, seja no ensino a distância. Dados do Ministério da Educação (MEC), que avaliou o período entre 2010 e 2014, indicaram uma taxa de evasão de 11,4\% em 2010, que aumentou para $49 \%$ em 2014. Tais resultados indicam que a evasão tem sido um fenômeno cada vez mais presente no âmbito acadêmico, reforçando a necessidade de compreender as motivações e os aspectos relacionados na decisão de abandonar um curso.

A evasão universitária pode ocorrer de formas distintas em termos de tipo de flutuação no sistema de ensino (Heublein, 2014). Pode ser entendida como o desligamento de um curso de nível superior por qualquer motivo que não seja sua conclusão (Castro \& Teixeira, 2014), relacionando-se com o curso escolhido (mudança do curso de origem, mas nem sempre de instituição), com a instituição (mudança de instituição, mas não necessariamente de curso) e com o sistema educacional (desistência da formação superior). Os motivos para que a pessoa se evada de um curso são diversos, como insatisfação com a escolha profissional, problemas financeiros e de saúde ou insatisfação e dificuldade de adaptação ao novo contexto. Considerando a multiplicidade de fatores relacionados à evasão, os investimentos das instituições de ensino superior (IES) em políticas educacionais têm crescido com o intuito de promover a permanência dos alunos nos cursos (Costa \& Dias, 2015).

Entre os potenciais motivos para a permanência ou evasão dos alunos nas IES, encontra-se a adaptação acadêmica. Tinto (1993) aponta que, para a transição e a adaptação ocorrerem com sucesso, é necessário que o aluno consiga realizar a integração tanto dos aspectos acadêmicos como dos sociais. Em suma, o aluno em um novo ambiente deve desenvolver estratégias que lhe permitam lidar com as novas experiências acadêmicas, como organização dos estudos e métodos de aprendizagem, e, ao mesmo 
tempo, adaptar-se ao convívio com pessoas diferentes das quais estava habituado. Em alguns casos, pode ser necessário lidar com mudanças de cidade, saída da casa dos pais, a necessidade de desenvolver autonomia e novos vínculos e, ao mesmo tempo, lidar com a escolha profissional realizada (Ogushi \& Bardagi, 2015). A instituição de ensino também pode colaborar na adaptação e transição ao incentivar a criação de espaços colaborativos para que os alunos compartilhem suas experiências, integrar a história pregressa do aluno na formação profissional e adotar estratégias de aprendizagem não limitadas ao ensino tradicional (Gale \& Parker, 2014).

Assim, a adaptação acadêmica no ES refere-se ao ajustamento do indivíduo a essa nova vida que se iniciou e está em curso (Oliveira, Carlotto, Vasconcelos, \& Dias, 2014). $O$ ingresso na universidade é uma fase complexa na vida do estudante, ainda mais se for jovem e suas vivências sociais até o momento forem escassas, visto que esse novo ambiente demanda habilidades que não são desenvolvidas e estimuladas na educação básica (Teixeira, Dias, Wottrich, \& Oliveira, 2008). Nessa etapa, o jovem passa a conviver com novas pessoas - colegas e professores - que poderão facilitar a sua adaptação acadêmica (Almeida \& Soares, 2003; Oliveira, Wiles, Fiorin, \& Dias, 2014). Questões relacionadas à realidade do ES podem causar dificuldades adaptativas, quando muda a constituição dos vínculos, já que o acadêmico pode não ter tanta proximidade e proteção que vivenciava no ensino médio (Bardagi \& Hutz, 2012), o que torna necessário um envolvimento mais ativo do estudante com a própria formação.

Devido a todas as variáveis citadas até o momento, o construto da adaptação à universidade e/ou vivência acadêmica vem sendo estudado há muito anos por meio do Questionário de Vivências Acadêmicas (QVA) elaborado por pesquisadores portugueses em 1999 e desde então aprimorado. A literatura que embasa o QVA aponta que a adaptação à universidade envolve os seguintes aspectos: estudo, carreira e aspectos pessoais e emocionais, interpessoais e institucionais. $O$ instrumento utilizado nesta pesquisa foi o Questionário de Adaptação ao Ensino Superior - QAES (Araújo et al., 2014), que segue o mesmo embasamento teórico do QVA, com cinco dimensões. Apesar de renomear os fatores, o QAES avalia os mesmos aspectos: projeto de carreira, adaptação social, adaptação pessoal emocional, adaptação ao estudo e adaptação institucional. Ressalta-se que o instrumento foi elaborado em parceria por pesquisadores portugueses e brasileiros, tendo assim um caráter multicultural.

É comum encontrar estudos que enfatizam as vivências acadêmicas tanto no cenário nacional como no internacional (Soares, Mourão, Santos, \& Mello, 2015), porém ainda são escassas as publicações que correlacionam a adaptação acadêmica a outros construtos psicológicos e utilizam o QAES. Um estudo com 217 estudantes do primeiro ano da Universidade de Columbia teve como objetivo verificar a relação entre adaptação acadêmica, personalidade, suporte social e inteligência emocional. Os resultados indicaram que características de personalidade, como extroversão, facilitam a interação social e garantem um apoio social significativo na adaptação acadêmica. Foram observadas ainda correlações positivas e com significância estatística entre a adaptação pessoal e emocional e a reparação do estado emocional $(r=0,37)$, entre a satisfação e o suporte 
social $(r=0,29)$ e uma correlação negativa com o neuroticismo $(r=-0,42)$. Além disso, constatou-se que a percepção do suporte social é uma variável importante no ajustamento dos estudantes ao ES (Tomás, Ferreira, Araújo, \& Almeida, 2014).

As experiências de sucesso e fracasso na integração de estudantes à universidade foram analisadas por Magalhães (2013) em um estudo qualitativo exploratório, em uma instituição privada do Sul do Brasil. Participaram 12 estudantes, divididos igualmente de acordo com escores indicativos de integração e indicativos de não integração à universidade. A análise de conteúdo dos depoimentos sugeriu que intenções de evasão dependem primariamente da frustração de expectativas sobre a vida universitária, já que as experiências acadêmicas e sociais mostraram impacto na certeza da escolha profissional e na consequente intenção de abandonar o curso. Os resultados evidenciaram ainda que a integração social é um fator importante para a satisfação e a permanência no curso escolhido.

O autor também identificou que o valor das mensalidades pode tornar-se um aspecto dificultador para integração, encontrando, assim, bastante relevância na adaptação acadêmica (Magalhães, 2013). Além disso, a renda influencia a própria possibilidade de realizar a escolha vocacional, bem como gera uma divisão social na qual universitários de famílias com melhores condições econômicas podem trocar de curso com uma frequência maior do que aqueles oriundos de famílias de baixa renda (Sampaio, Sampaio, Mello, \& Melo, 2011). Outro impacto da renda está relacionado ao tipo de formação recebido durante os ciclos fundamental e médio, já que indivíduos com maior renda podem frequentar cursos preparatórios e dedicar-se integralmente ao estudo, fato que pode não ocorrer com pessoas de baixa renda, ocasionando dificuldade para acompanhar o novo estilo de aprendizagem no ES (Kückelhaus, Santos, \& Luz, 2017). Assim, pode-se afirmar que as condições financeiras têm participação importante na experiência universitária de estudantes, e, de acordo com o nível socioeconômico, podem ser desfavoráveis, dificultando assim a adaptação.

Considerando esses apontamentos sobre as variáveis que contribuem para a adaptação acadêmica, acredita-se que, quando a adaptação não é favorável ao aluno, aumenta-se a probabilidade de que ele se evada do curso escolhido. Além da dificuldade na adaptação, outros motivos podem contribuir para que o abandono do curso ocorra, como as expectativas dos estudantes acerca de sua carreira no futuro (Ambiel, Santos, \& Dalbosco, 2016), dificuldades financeiras para trabalhar e pagar a faculdade, descontentamento com questões institucionais, reprovas ou mau desempenho acadêmico e falta de informação sobre as profissões e o curso (Bardagi \& Hutz, 2005).

Além das questões elencadas, o aspecto vocacional aparece como um fator determinante para a permanência ou evasão universitária. No âmbito vocacional, encontram-se a escolha profissional e o projeto de carreira, entendidos como o planejamento e a integração dos interesses profissionais, valores, habilidades e o lugar que se deseja ocupar no mundo laboral (Bohoslavsky, 1977). Bardagi e Hutz (2005) indicam que há uma estrita ligação entre a qualidade da escolha e a construção da trajetória profissional. Desse modo, a evasão em função da identidade vocacional pode ser 
compreendida de duas maneiras, sendo a primeira uma medida relativamente tardia de exploração profissional, em que o aluno abandona um curso e inicia outro ou muda de área sem a necessidade de um curso de nível superior; a segunda é o amadurecimento das preferências e dos projetos vocacionais, que pode eliminar a indecisão e acarretar em melhor clareza do projeto de vida, levando o aluno a abandonar um curso e iniciar outro (Araújo \& Sarriera, 2004).

Ante o exposto, é possível afirmar que a evasão é um fenômeno social complexo que vem preocupando as instituições de ensino, pois a saída de alunos provoca graves consequências sociais, acadêmicas e econômicas. São poucas as pesquisas sobre a temática, assim como são raras as instituições que possuem um programa institucional regular de combate à evasão, com planejamento de ações, acompanhamento de resultados e coleta de experiências bem-sucedidas (Silva Filho, Motejunas, Hipólito, \& Lobo, 2007). Cabe ressaltar que tal fenômeno não é restrito apenas ao contexto brasileiro, sendo pautado por pesquisadores de outros países (Arce, Crespo, \& Míguez-Álvarez, 2015; Ćukušić, Garača, \& Jadrić, 2014; Hublein, 2014) interessados em compreender as complexidades e particularidades culturais envolvidas na evasão universitária.

Nesse sentido, o presente estudo, de caráter exploratório, tem como objetivo verificar correlações entre a adaptação acadêmica e os motivos para evasão do ensino superior por meio da Escala de Motivos para Evasão no Ensino Superior (M-ES) e do QAES, controlando (correlação parcial) com satisfação com o curso e com a renda atribuída pelos participantes. Levantou-se como hipóteses que pessoas com maiores médias no QAES apresentarão menores pontuações em "motivos relacionados à carreira" e "motivos vocacionais" como razão para evadir-se do curso. No que se refere à correlação parcial entre satisfação com a escolha profissional e renda mensal, espera-se que a magnitude das correlações mude expressivamente quando comparadas às correlações sem controle, fornecendo melhores indicativos sobre a influência das variáveis em relação à evasão universitária.

\section{Método}

\section{Participantes}

Participaram deste estudo 198 estudantes universitários, sendo 29 do sexo masculino $(14,6 \%)$ e 169 do sexo feminino $(85,4 \%)$, com idades variando entre 18 e 52 anos $(M=20,92$, DP $=4,66)$. Sobre o tipo de instituição, 55,6\% ( $N=110)$ declararam estudar em universidades públicas e $44,4 \%$ em universidades particulares $(N=88)$, dispersos em nove semestres, com maior concentração nos três semestres iniciais (51\%). Em relação ao turno de estudo, predominaram alunos do período noturno $(41,9 \%)$, seguidos daqueles do matutino (23,7\%), integral $(27,3 \%)$ e vespertino $(7,1 \%)$. No tocante aos cursos, foram registrados 67 cursos diferentes, e as maiores frequências foram de alunos de Psicologia (20,2\%), seguidos de Direito $(6,1 \%)$ e Administração $(5,6 \%)$, e as menores frequências foram registradas para cursos como Zootecnia $(0,5 \%)$, Hotelaria $(0,5 \%)$ e Relações Públicas $(0,5 \%)$. 
Os participantes foram oriundos de 21 estados do Brasil, com maior quantidade em São Paulo ( $N=39,19,7 \%)$ e Rio Grande do Sul ( $N=30,15,2 \%)$ e menor número nos estados de Sergipe e Tocantins, com apenas um participante em cada (0,5\%). Acerca da renda mensal, 51,4\% preferiram não responder, e 32,6\% declararam estar na faixa de $R \$ 1.760$ a $R \$ 4$ 4.399. As menores concentrações de pessoas estiveram na faixa de $R \$ 4.400$ a $R \$ 6.159(8,8 \%), R \$ 6.160$ a $R \$ 8.799(2,8 \%)$ e acima de $R \$ 8.000$ (4,4\%). Trata-se de uma amostra não probabilística.

\section{Instrumentos}

Questionário sociodemográfico: com questões de caracterização da amostra relativas a idade, sexo, curso, tipo de instituição, renda, período de estudo, cidade e estado.

Escala de Motivos para Evasão no Ensino Superior - M-ES (Ambiel, 2017): com 53 itens, esse instrumento tem como objetivo avaliar potenciais motivos para que o aluno se evada do curso superior. Participaram dos estudos de normatização 1.557 estudantes universitários, sendo 757 do sexo masculino $(48,6 \%)$ e 797 do feminino $(51,2 \%)$, com idade média de 23,5 anos. Estudos de análise fatorial evidenciaram sete fatores assim denominados: motivos institucionais, vocacionais, relacionados à falta de suporte, relacionados à carreira, relacionados ao desempenho acadêmico, interpessoais e relacionados à autonomia. As cargas fatoriais dos itens variaram de 0,33 a 0,81, e os índices de precisão dos fatores (alfa de Cronbach) foram entre adequados e excelentes, com valores entre 0,74 e 0,90.

Questionário de Adaptação ao Ensino Superior - QAES (Araújo et al., 2014): esse instrumento contém 40 itens que avaliam cinco dimensões: projeto de carreira, adaptação social, adaptação pessoal emocional, adaptação ao estudo e adaptação institucional. Nos estudos psicométricos, apresentou coeficientes de precisão que variaram entre 0,72 para o fator adaptação institucional e 0,92 para o fator adaptação pessoal-emocional. O instrumento é respondido em uma escala de resposta Likert que varia entre 1 ("discordo totalmente") e 5 ("concordo totalmente").

\section{Procedimentos}

A pesquisa foi autorizada pelo Comitê de Ética em Pesquisa da Universidade São Francisco, e as coletas ocorreram de forma on-line por meio da plataforma Google Forms, de março a maio de 2016. Os participantes deveriam concordar com o Termo de Consentimento Livre e Esclarecido para acessarem os questionários. As respostas aos instrumentos levaram aproximadamente 20 minutos.

\section{Análise de dados}

Os dados foram analisados por meio do software Statistical Package for the Social Sciences (SPSS) versão 21, com estatísticas descritivas para caracterização da amostra. Posteriormente, ocorreram as estatísticas inferenciais com análise de correlação de 
Pearson entre os fatores dos instrumentos e com controle de variáveis por meio da correlação parcial.

\section{Resultados}

Inicialmente, foram calculadas as médias e os desvios-padrão das respostas aos instrumentos, e as maiores médias foram obtidas no fator "motivos relacionados à falta de suporte" da M-ES e no fator "projeto de carreira" do QAES. Na sequência, verificaram-se os índices de confiabilidade dos instrumentos com a amostra por meio do cálculo do alfa de Cronbach, resultando em índices de aceitáveis a elevados. Após esses procedimentos, realizou-se a correlação entre os fatores da M-ES e do QAES, tal como apresentado na Tabela 1.

\section{Tabela I. Correlações entre os fatores da M-ES e do QAES, médias e desvios- -padrão nos fatores e precisão.}

\begin{tabular}{|c|c|c|c|c|c|c|c|c|c|c|c|c|c|c|}
\hline & $M$ & DP & 1 & 2 & 3 & 4 & 5 & 6 & 7 & 8 & 9 & 10 & 11 & 12 \\
\hline I & 27,39 & 10,0 & $\begin{array}{l}\alpha= \\
0,90\end{array}$ & & & & & & & & & & & \\
\hline 2 & 24,03 & 8,32 & $0,43 * *$ & $\begin{array}{l}\alpha= \\
0,88\end{array}$ & & & & & & & & & & \\
\hline 3 & 30,10 & 9,66 & $0,53 * *$ & $0,53 * *$ & $\begin{array}{l}\alpha= \\
0,87\end{array}$ & & & & & & & & & \\
\hline 4 & 16,27 & 5,79 & $0,44 * *$ & $0,62 * *$ & $0,52 * *$ & $\begin{array}{l}\alpha= \\
0,83\end{array}$ & & & & & & & & \\
\hline 5 & 15,69 & 6,19 & $0,42 * *$ & $0,48 * *$ & $0,45^{* *}$ & $0,48 * *$ & $\begin{array}{l}\alpha= \\
0,86\end{array}$ & & & & & & & \\
\hline 6 & 13,98 & 5,50 & $0,5 I^{* * *}$ & $0,36 * *$ & $0,4 I^{* * *}$ & $0,54 * *$ & $0,47^{* * *}$ & $\begin{array}{l}\alpha= \\
0,86\end{array}$ & & & & & & \\
\hline 7 & 10,35 & 4,62 & $0,37 * *$ & $0,29 * *$ & $0,48 * *$ & $0,37 * *$ & $0,33^{* * *}$ & $0,40 * *$ & $\begin{array}{l}\alpha= \\
0,77\end{array}$ & & & & & \\
\hline 8 & 31,50 & 6,93 & $-0,64$ & $-0,26 * *$ & $-0,01$ & $-0,33^{*} *$ & $-0,18 * *$ & $-0,27^{* * *}$ & $-0,16$ & $\begin{array}{l}\alpha= \\
0,90\end{array}$ & & & & \\
\hline 9 & 30,26 & 7,37 & $-0,07$ & $-0,08$ & $-0,02$ & $-0,15 * *$ & $-0,17^{*}$ & $-0,37^{* * *}$ & $-0,08$ & $0,28 * *$ & $\begin{array}{l}\alpha= \\
0,92\end{array}$ & & & \\
\hline 10 & $|9,6|$ & 7,53 & $-0,13$ & $-0,11$ & $-0,06$ & $-0,27^{* *}$ & $-0,25 * *$ & $-0,3 \mid * *$ & $-0,07$ & $0,32 * *$ & $0,29 * *$ & $\begin{array}{l}\alpha= \\
0,87\end{array}$ & & \\
\hline 11 & 26,42 & 6,16 & $-0,12$ & $-0,21$ & $-0,10$ & $-0,26 * *$ & $-0,39 * *$ & $-0,27^{* * *}$ & $-0,20 * *$ & $0,43 * *$ & $0,35 * *$ & $0,39 * *$ & $\begin{array}{l}\alpha= \\
0,84\end{array}$ & \\
\hline 12 & 28,18 & 6,04 & $-0,12$ & 0,11 & $-0,01$ & $-0,01$ & 0,03 & $-0,16^{*}$ & $-0,81$ & $0,26 * *$ & $0,36 * *$ & 0,13 & $0,21 * *$ & $\begin{array}{l}\alpha= \\
0,78\end{array}$ \\
\hline
\end{tabular}

* Correlação significativa em nível de $p<0,05$; ** correlação significativa em nível de $p<0,0$ I.

$\mathrm{I}=$ motivos institucionais; 2 = motivos vocacionais; 3 = motivos relacionados à falta de suporte; $4=$ motivos relacionados à carreira; 5 = motivos relacionados ao desempenho acadêmico; $6=$ motivos interpessoais; $7=$ motivos relacionados à autonomia; 8 = projeto de carreira; 9 = adaptação social; 10 = adaptação pessoal-emocional; II = adaptação ao estudo; 12 = adaptação institucional.

Fonte: Elaborada pelos autores. 
Como observado na Tabela 1, obtiveram as maiores médias as dimensões "projeto de carreira" e "adaptação social", ambas do QAES. A maioria das correlações entre os fatores da M-ES e os fatores do QAES variaram de intensidade fraca a forte e com significância estatística. Os índices de confiabilidade na M-ES variaram de 0,77 a 0,90, ou seja, de baixo a moderado-elevado (Murphy \& Davidsholder, 1988). No QAES, os coeficientes ficaram na mesma faixa de classificação, com valores entre 0,78 e 0,92. Na sequência, foram realizadas correlações parciais entre os fatores dos instrumentos com a variável satisfação com a escolha da profissão como controle, cujos resultados podem ser vistos na Tabela 2:

\section{Tabela 2. Correlações parciais entre os fatores da M-ES e do QAES com controle da variável satisfação com a escolha da profissão.}

\begin{tabular}{|c|c|c|c|c|c|}
\hline & $\begin{array}{l}\text { Projeto de } \\
\text { carreira }\end{array}$ & $\begin{array}{l}\text { Adaptação } \\
\text { social }\end{array}$ & $\begin{array}{l}\text { Adaptação } \\
\text { emocional }\end{array}$ & $\begin{array}{l}\text { Adaptação } \\
\text { ao estudo }\end{array}$ & $\begin{array}{l}\text { Adaptação } \\
\text { institucional }\end{array}$ \\
\hline Motivos institucionais & $-0,00$ & $-0,06$ & $-0,12$ & $-0,10$ & $-0,11$ \\
\hline Motivos vocacionais & $-0,14 *$ & $-0,04$ & $-0,08$ & $-0,17 *$ & $0,14 *$ \\
\hline $\begin{array}{l}\text { Motivos relacionados à falta } \\
\text { de suporte }\end{array}$ & $-0,01$ & $-0,02$ & $-0,05$ & $-0,10$ & $-0,01$ \\
\hline Motivos relacionados à carreira & $-0,27^{* *}$ & $-0,13$ & $-0,25 * *$ & $-0,21 * *$ & $-0,01$ \\
\hline $\begin{array}{l}\text { Motivos relacionados ao } \\
\text { desempenho acadêmico }\end{array}$ & $-0,08$ & $-0,14 *$ & $-0,23 * *$ & $-0,36 * *$ & 0,05 \\
\hline Motivos interpessoais & $-0,23 * *$ & $-0,35 * *$ & $-0,29 * *$ & $-0,24 * *$ & $-0,15^{*}$ \\
\hline $\begin{array}{l}\text { Motivos relacionados } \\
\text { à autonomia }\end{array}$ & 0,06 & $-0,09$ & $-0,08$ & $-0,21 * * *$ & $-0,08$ \\
\hline
\end{tabular}

Fonte: Elaborada pelos autores.

A maior parte das correlações obtidas não foi significativa estatisticamente ou teve coeficientes nulos. Entre as que obtiveram significância estatística, todas foram no sentido negativo e com magnitude variando de fraca a moderada. Entretanto, quando se controlou a variável satisfação com a escolha da profissão, percebeu-se uma diminuição expressiva no coeficiente de correlação entre "motivos vocacionais" e "projeto de carreira", "motivos relacionados à carreira" e "adaptação ao estudo", e entre "motivos relacionados à autonomia" e "adaptação Institucional". Por fim, realizaram-se correlações parciais entre os fatores da M-ES e do QAES, tendo como critério de controle a variável renda mensal. Para essa análise, consideraram-se apenas os participantes que informaram a renda mensal $(48,1 \%)$. A Tabela 3 apresenta as correlações obtidas entre os fatores dos instrumentos: 
Tabela 3. Correlações parciais entre os fatores da M-ES e do QAES com controle da variável renda mensal.

\begin{tabular}{|c|c|c|c|c|c|}
\hline & $\begin{array}{l}\text { Projeto de } \\
\text { carreira }\end{array}$ & $\begin{array}{l}\text { Adaptação } \\
\text { social }\end{array}$ & $\begin{array}{l}\text { Adaptação } \\
\text { emocional }\end{array}$ & $\begin{array}{l}\text { Adaptação } \\
\text { ao estudo }\end{array}$ & $\begin{array}{l}\text { Adaptação } \\
\text { institucional }\end{array}$ \\
\hline Motivos institucionais & $-0,07$ & $-0,07$ & $-0,11$ & $-0,12$ & $-0,12$ \\
\hline Motivos vocacionais & $-0,27^{* * *}$ & $-0,10$ & $-0,09$ & $-0,23 *$ & 0,10 \\
\hline $\begin{array}{l}\text { Motivos relacionados à falta } \\
\text { de suporte }\end{array}$ & 0,00 & $-0,02$ & $-0,02$ & $-0,10$ & $-0,03$ \\
\hline Motivos relacionados à carreira & $-0,33^{*} *$ & $-0,15 *$ & $-0,25 * *$ & $-0,25 * *$ & $-0,00$ \\
\hline $\begin{array}{l}\text { Motivos relacionados ao } \\
\text { desempenho acadêmico }\end{array}$ & $-0,17 *$ & $-0,17 *$ & $-0,26 * *$ & $-0,39 * *$ & 0,02 \\
\hline Motivos interpessoais & $-0,28 * *$ & $-0,35 * *$ & $-0,30 * *$ & $-0,29 * *$ & $-0,16 *$ \\
\hline $\begin{array}{l}\text { Motivos relacionados à } \\
\text { autonomia }\end{array}$ & 0,02 & $-0,05$ & $-0,05$ & $-0,19 * *$ & $-0,06$ \\
\hline
\end{tabular}

Legenda: * $\mathrm{p}>0,05 ;{ }^{*} * \mathrm{p}<0,00 \mathrm{I}$.

Fonte: Elaborada pelos autores.

Como observado na Tabela 3, apenas três correlações foram positivas, porém com valores nulos. Todas as demais foram negativas com intensidades entre baixa e moderada, além de a maior parte dos resultados não atingir significância estatística. Percebe-se que, após a inserção da variável de controle, houve diminuição expressiva nos coeficientes de correlação entre os fatores "motivos institucionais" e "projeto de carreira", "motivos relacionados à falta de suporte" e "adaptação emocional", e "motivos relacionados à autonomia" e "adaptação institucional". Em contrapartida, houve aumento nos coeficientes entre "motivos vocacionais", "motivos interpessoais" e "adaptação ao estudo".

\section{Discussão e conclusão}

Considerando o alto índice de evasão universitária no Brasil (Ministério da Educação, 2013) e a importância da compreensão da temática para as IES (Costa \& Dias, 2015), bem como para o avanço científico da área, este trabalho teve o objetivo de verificar correlações entre a adaptação acadêmica e os motivos para evasão do ensino superior por meio da M-ES e do QAES e pelo controle das variáveis satisfação com a escolha profissional e renda atribuída pelos participantes. Especificamente sobre as propriedades psicométricas dos instrumentos, pode-se observar que os índices de precisão tiveram um leve aumento quando comparados com a amostra normativa em que os valores ficaram entre 0,74 e 0,90 na M-ES (Ambiel, 2017) e entre 0,78 e 0,92 no QAES.

Acerca das correlações entre os instrumentos (Tabela 1), verificou-se que o fator "motivos relacionados à carreira" M-ES correlacionou-se significativamente com todos 
os demais fatores dos dois instrumentos, corroborando a importância dos aspectos vocacionais e de projeto de vida como fatores potenciais para a evasão (Bardagi \& Hutz, 2005). Por meio dos resultados, percebe-se que as pessoas que têm maior clareza do seu projeto de carreira profissional tendem a apresentar menores motivos para evadir-se do curso em função da insatisfação com a escolha e que essa segurança na carreira pode estar relacionada a atitudes autônomas e ativas ante o papel de aluno, como sugere a correlação positiva desse fator com "motivos relacionados à autonomia". Assim, quanto mais adaptado o aluno estiver ao curso, nos aspectos emocionais e sociais e à instituição, menores serão as chances de evasão por "motivos relacionados à carreira".

O fator "motivos interpessoais" da M-ES também obteve correlações estatisticamente significativas com todos os demais fatores dos instrumentos utilizados. O resultado converge na direção dos apontamentos de Soares et al. (2014), em que os alunos que se envolvem mais na formação de vínculos afetivos seguros na relação com os colegas tendem a adaptar-se melhor ao contexto. As correlações negativas entre esse fator e as dimensões do QAES e a correlação obtida com a dimensão social indica que quanto maior for a dificuldade em estabelecer vínculos no ambiente acadêmico, maiores serão as chances de o aluno abandonar curso por falta de interação com os colegas. Assim, é importante que as IES promovam meios para que ocorra a integração entre os alunos e todas as demais pessoas envolvidas na área (coordenação, professores, funcionários), uma vez que elas têm papel fundamental para que o aluno crie senso de pertencimento à instituição e consiga adaptar-se de maneira mais fácil (Almeida \& Soares, 2003; Oliveira et al., 2014).

No que se refere à correlação parcial com satisfação com a escolha profissional, percebeu-se um aumento nos coeficientes entre "motivos vocacionais", "projeto de carreira" e "adaptação ao estudo", e entre "motivos relacionados à autonomia" e "adaptação institucional". Os resultados indicam que estudantes mais satisfeitos com a escolha tendem a ter maior organização e adaptação ao novo formato de ensino-aprendizagem, o que pode gerar um melhor desempenho e minimizar as chances de evasão por esse motivo. Desse modo, concorda-se com Ogushi e Bardagi (2015) que os trabalhos de orientação de carreira devem estar disponíveis na transição e no período intermediário da graduação, de modo a contribuir, ao longo de toda a trajetória acadêmica, para que o aluno reflita sobre sua escolha profissional inicial e sobre todas as etapas que surgirem no decorrer do curso (estágios, ênfases de formação, áreas de atuação). Dessa forma, ele será capaz de adaptar-se positivamente aos novos cenários que não se limitam à transição do ensino médio para o superior.

Por fim, na correlação parcial com a variável renda, ainda que com a limitação da amostra nessa análise, foi possível constatar uma diminuição nos coeficientes entre "motivos institucionais" e "projeto de carreira", "motivos relacionados à falta de suporte" e "adaptação emocional", e "motivos relacionados à autonomia, e "adaptação institucional", o que sugere que a renda pode influenciar a adaptação dos alunos ao ensino superior e potencializar ou minimizar os motivos para evasão, especificamente 
quando se refere ao suporte financeiro e social para a adaptação universitária. Desse modo, para além do delineamento adequado do plano de carreira ou das relações interpessoais estabelecidas no contexto acadêmico, é preciso destacar as implicações do nível socioeconômico para permanência no ensino superior e adaptação a ele (Magalhães, 2013).

Conforme apontado inicialmente, as políticas públicas de acesso ao ensino superior dos últimos anos permitiram o ingresso de populações de nível socioeconômico baixo nas universidades (Ministério da Educação, 2013). Todavia, para além da possibilidade de iniciar um curso, é necessário que haja meios de favorecer a permanência dessas populações nas IES, uma vez que a renda mensal é uma das variáveis intervenientes para adaptação dos alunos, conforme visto nos resultados deste estudo. Assim, questões como valores de mensalidade (Magalhães, 2013) ou outros custos oriundos dos cursos e situação de alunos bolsistas (Zago, 2006) ou cotistas devem ser elementos considerados em estudos sobre adaptação e evasão acadêmica.

Esta pesquisa atingiu seus objetivos e possibilitou expandir o conhecimento no campo de investigações sobre evasão e adaptação acadêmica, aspectos ainda pouco investigados na literatura. $O$ estudo apresenta limitações na composição da amostra que não é equilibrada em função do sexo dos participantes, uma vez que houve predominância do sexo feminino. Também há uma quantidade vasta de estados sem equilíbrio na distribuição dos participantes, com pouca representatividade em grande parte. Além disso, embora tenham sido realizadas análises com a variável renda, não foram abordados se os estudantes eram bolsistas ou cotistas, fato que poderia ampliar as discussões sobre a temática e colaborar para a compreensão do fenômeno de evasão e adaptação com esses grupos. Outro elemento limitador dos resultados referentes à renda está relacionado ao fato de grande parte da amostra não ter fornecido essa informação. Assim, sugerem-se pesquisas que contemplem essa variável e com melhor estratificação para verificar diferença em função do nível socioeconômico.

Compreender a multiplicidade de fatores envolvidos no processo de adaptação acadêmica pode colaborar para as IES, públicas ou privadas, desenvolverem programas que visem auxiliar os estudantes na transição do ensino médio ao superior, assim como durante todas as eventuais necessidades de adaptação ao longo da formação (Ogushi \& Bardagi, 2015). À medida que essas variáveis forem exploradas, compreendidas e resultarem em medidas facilitadoras para os alunos, consequentemente, a evasão decorrente da inadaptação será minimizada e o envolvimento do estudante com seu curso poderá se tornar mais proveitoso.

\section{Referências}

Almeida, L. S., \& Soares, A. P. (2003). Os estudantes universitários: sucesso escolar e desenvolvimento psicossocial. In: E. Mercuri, \& S. A. J. Polydoro (Orgs.), Estudante universitário: características e experiências de formação. Taubaté: Cabral. 
Ambiel, R. A. M. (2017). Escala de Motivos para Evasão do Ensino Superior: manual técnico. São Paulo: Hogrefe.

Ambiel, R. A. M., Santos, A. A. A. D., \& Dalbosco, S. N. P. (2016). Motivos para evasão, vivências acadêmicas e adaptabilidade de carreira em universitários. Psico, 47(4), 288-297. doi:10.15448/1980-8623.2016.4.23872

Araújo, A. M., Almeida, L. S., Ferreira, J. A., Santos, A. A., Noronha, A. P., \& Zanon, C. (2014). Questionário de Adaptação ao Ensino Superior (QAES): construção e validação de um novo questionário. Psicologia, Educação e Cultura, XVIII(1), 131-145.

Araújo, J. S., \& Sarriera, J. C. (2004). Redirecionamento da carreira profissional: uma análise compreensiva. In J. C. Sarriera, K. B. Rocha, \& A. Pizinato (Orgs.), Desafios do mundo do trabalho: orientação, inserção e mudanças. Porto Alegre: EDIPUCRS.

Arce, M. E., Crespo, B., \& Míguez-Álvarez, C. (2015). Higher education drop-out in spain-particular case of universities in Galicia. International Education Studies, 8(5), 247-264. doi:10.5539/ies.v8n5p247

Bardagi, M. P., \& Hutz, C. S. (2005). Evasão e serviços de apoio ao estudante: uma breve revisão da literatura brasileira. Psicologia Revista, 14(2), 279-301. Recuperado em 26 Junho, 2018, de https://revistas.pucsp.br/index.php/psicorevista/article/ view/18107

Bardagi, M. P., \& Hutz, C. S. (2012). Rotina acadêmica e relação com colegas e professores: impacto na evasão universitária. Psico, 43(2), 174-184. Recuperado em 21 maio, 2018, de http://revistaseletronicas.pucrs.br/teo/ojs/index.php/revistapsico/ article/view/7870/8034

Bohoslavsky, R. (1977). Orientação vocacional: a estratégia clínica. São Paulo: Martins Fontes.

Castro, A. K. S. S., \& Teixeira, M. A. (2014). Evasão universitária: modelos teóricos internacionais e o panorama das pesquisas no Brasil. Psicologia Argumento, 32(79), 9-17. doi:10.7213/psicol..argum.32.s02.A001

Costa, S. L., \& Dias, S. M. B. (2015). A permanência no ensino superior e as estratégias institucionais de enfrentamento da evasão. Jornal de Políticas Educacionais, 9(17-18), 51-60. doi:10.5380/jpe.v9i17/18.38650

Ćukušić, M., Garača, Ž., \& Jadrić, M. (2014). Online self-assessment and students' success in higher education institutions. Computers \& Education, 72, 100-109. doi:10.1016/j.compedu.2013.10.018

Gale, T., \& Parker, S. (2014). Navigating change: a typology of student transition in higher education. Studies in Higher Education, 39(5), 734-753. doi:10.1080/0307 5079.2012.721351

Heublein, U. (2014). Student drop-out from german higher education institutions. European Journal of Education, 49(4), 497-513. doi:10.1111/ejed.12097 
Kückelhaus, S. D. S. G. P., dos Santos, A. P. C., \& Luz, C. N. M. (2017). Evasão universitária do curso de administração da faculdade itop. Multidebates, 1(1), 8-27. Recuperado em 21 maio, 2018, de http://itopedu.com.br/revista/index.php/revista/ article/view/3/2

Magalhães, M. O. (2013). Sucesso e fracasso na integração do estudante à universidade: um estudo comparativo. Revista Brasileira de Orientação Profissional, 14(2) 215-226. Recuperado em 21 maio, 2018, de http://pepsic.bvsalud.org/pdf/ rbop/v14n2/07.pdf

Ministério da Educação (2013). Desenvolvimento, aprimoramento e consolidação de uma educação nacional de qualidade. Brasília: Conselho Nacional de Educação. Recuperado em 21 maio, 2018, de http://portal.mec.gov.br/

Murphy, K., \& Davidsholder, C. (1988). Psychological testing: Principles and applications. Englewood Cliffs, New Jersey: Prentice Hall.

Ogushi, M. M. P., \& Bardagi, M. P. (2015). Reflexões sobre a relação estudante-universidade a partir de uma experiência de atendimento em orientação profissional. Extensio: Revista Eletrônica de Extensão, 12(19), 33-50. doi:10.5007/1807$-0221.2015 \mathrm{v} 12 \mathrm{n} 19 \mathrm{p} 33$

Oliveira, C. T., Carlotto, R. C., Vasconcelos, S. J. L., \& Dias, A. C. G. (2014). Adaptação acadêmica e coping em estudantes universitários brasileiros: uma revisão de literatura. Revista Brasileira de Orientação Profissional, 15(2), 177-186. Recuperado em 21 maio, 2018, de http://pepsic.bvsalud.org/pdf/rbop/v15n2/08.pdf

Oliveira, C. T., Wiles, J. M., Fiorin, P. C., \& Dias, A. C. G. (2014). Percepção de estudantes universitários sobre a relação professor-aluno. Revista Quadrimestral da Associação Brasileira de Psicologia Escolar e Educacional, 18(2) 239-246. doi:10.15 90/2175-3539/2014/0182739

Sampaio, B., Sampaio, Y., de Mello, E. P., \& Melo, A. S. (2011). Desempenho no vestibular, background familiar e evasão: evidências da UFPE. Economia Aplicada, 15(2), 287-309. doi:10.1590/S1413-80502011000200006

Silva Filho, R. L. L., Motejunas, P. R., Hipólito, O., \& Lobo, M. B. C. M. (2007). A evasão no ensino superior brasileiro. Cadernos de Pesquisas, 37(132). doi:10.15 90/S0100-15742007000300007

Soares, A. B., Francischetto, V., Dutra, B. M., Miranda, J. M. D., Nogueira, C. C. D. C., Leme, V. R., ... \& Almeida, L. S. (2014). O impacto das expectativas na adaptação acadêmica dos estudantes no ensino superior. Psico-USF, 19(1), 49-60. Recuperado em 21 maio, 2018, de http://www.redalyc.org/pdf/4010/401041441006.pdf

Soares, A. B., Mourão, L., Santos, A. A. A., \& Mello, T. V. S. (2015). Habilidades sociais e vivência acadêmica de estudantes universitários. Interação em Psicologia, 19(2), 211-223. 
Teixeira, M. A. P., Dias, A. C. G., Wottrich, S. H., \& Oliveira, A. M. (2008). Adaptação à universidade em jovens calouros. Revista Semestral da Associação Brasileira de Psicologia Escolar e Educacional, 12(1), 185-202. doi:10.1590/\$1413-85572008000 100013

Tinto, V. (1993). Leaving college: rethinking the causes and cures of student attrition. Chicago: University of Chicago Press.

Tomás, R. A., Ferreira, J. A., Araújo, A. M., \& Almeida, L. S. (2014) Adaptação pessoal e emocional em contexto universitário: o contributo da personalidade, suporte social e inteligência emocional. Revista Portuguesa de Pedagogia, 48(2), 87-107 doi:10.14195/1647-8614_48-2_5

Zago, N. (2006). Do acesso à permanência no ensino superior: percursos de estudantes universitários de camadas populares camadas populares. Revista Brasileira de Educação, 11(32), 227. Recuperado em 21 maio, 2018, de http://www.scielo. $\mathrm{br} / \mathrm{pdf} / \% 0 \mathrm{D} / \mathrm{rbedu} / \mathrm{v} 11 \mathrm{n} 32 / \mathrm{a} 03 \mathrm{v} 11 \mathrm{n} 32 . \mathrm{pdf}$

Submissão: 13.3.2017

Aceite: 4.4 .2018 